\title{
ON THE SPATIAL ASYMPTOTIC BEHAVIOR OF STOCHASTIC FLOWS IN EUCLIDEAN SPACE
}

\author{
By Peter Imkeller ANd Michael Scheutzow \\ Humboldt-Universität and Technische Universität Berlin
}

\begin{abstract}
We study asymptotic growth rates of stochastic flows on $\mathbf{R}^{d}$ and their derivatives with respect to the spatial parameter under Lipschitz conditions on the local characteristics of the generating semimartingales. In a first step these conditions are seen to imply moment inequalities for the flow $\phi$ of the form$$
E \sup _{0 \leq t \leq T}\left|\phi_{0 t}(x)-\phi_{0 t}(y)\right|^{p} \leq|x-y|^{p} \exp \left(c p^{2}\right) \quad \text { for all } p \geq 1 .
$$

In a second step we deduce the growth rates from an integrated version of these moment inequalities, using the continuity lemma of Garsia, Rodemich and Rumsey. We provide two examples to show that our results are sharp.
\end{abstract}

0. Introduction. Stochastic differential equations generated by sufficiently smooth vector fields on $\mathbf{R}^{d}$ are known to generate flows of homeomorphisms or diffeomorphisms of $\mathbf{R}^{d}$. For an account of this and the main facts about stochastic flows, see [5]. In the deterministic setting, any flow generated by vector fields with at most linear growth in the spatial parameter has sublinear growth as well. This is no longer true in the presence of semimartingale noise driving the stochastic differential equations which generate the flow.

Their a.s. spatial asymptotic growth rate has been studied in [11], [5], pages 163,176 and [10]. In the first two papers, it is shown that for $|x| \rightarrow \infty$ the supremum over $s, t$ in a compact interval of the modulus of the flow $\phi_{s t}(x)$ grows at most like $|x|^{1+\varepsilon}$ and at least like $|x|^{1-\varepsilon}$ for any $\varepsilon>0$, and similar results hold for the derivative of the flow and its inverse in case it consists of diffeomorphisms. Ocone and Pardoux [11] consider stochastic differential equations driven by finite-dimensional Brownian motion, whereas Kunita [5] works in a more general class of driving semimartingales. In Kunita's [5] general setting, Mohammed and Scheutzow [10] improve these results by showing that the growth rate of the flow at infinity can be at most $|x|(\ln |x|)^{\varepsilon}$, for any positive $\varepsilon$.

Our motivation for writing this paper comes from the same source as Mohammed and Scheutzow's: the global a.s. rates of spatial growth of flows will play a role in questions of existence of infinite-dimensional flows associated with quasilinear stochastic hereditary systems and stochastic partial differential equations (see [7], [8], [9]). Looking at the results of [10], we asked: can we obtain exact rates of growth by applying the lemma of Garsia, Rodemich

Received August 1997; revised May 1998.

AMS 1991 subject classifications. Primary 60H10, 34F05; secondary 60G48, 60G17.

Key words and phrases. Stochastic differential equation, stochastic flow, spatial growth rate, modulus of continuity, GRR lemma, semimartingale. 
and Rumsey in its majorizing measure version to moment inequalities for the flow, while carefully keeping track of the quality of the constants appearing in the main martingale inequalities of Doob and Burkholder, Davis and Gundy? It is the main task of this paper to show that the answer to this question is "yes."

The paper is organized as follows. In the first section we review the $m a$ jorizing measure form of the GRR lemma. In Theorems 1.1, 1.2 and 1.3, this key lemma is used to derive moduli of continuity for random fields on $\mathbf{R}^{d}$ with values in some metric space $(M, \rho)$ which satisfy moment conditions of the form

$$
E\left[\rho(\phi(x), \phi(y))^{p}\right] \leq d(x, y)^{p} \exp \left(c p^{2}\right),
$$

either for some fixed $p$ and the metric $d(x, y)=|x-y|$ (Theorem 1.1), for all $p \geq 1$ and the metric $d(x, y)=|x-y|$ (Theorem 1.2) or for all $p \geq 1$ and the metric $d(x, y)=|x-y| \wedge 1, x, y \in M$ (Theorem 1.3).

In Section 2 we verify the conditions of Theorem 1.2 for the stochastic flow $\phi$ generated by a stochastic differential equation driven by a semimartingale $F$ whose local characteristics satisfy suitable Lipschitz conditions. Together with a similar estimate on the inverse of the modulus of the flow (Proposition 2.2) we obtain Theorem 2.1, which shows that the supremum of $\left|\phi_{s t}(x)\right|$ over $0 \leq s$, $t \leq T$ is bounded by a random variable $Y$ multiplied by $|x| \exp \left(\gamma(\ln \ln |x|)^{1 / 2}\right)$ as $|x| \rightarrow \infty$ for some constant $\gamma>0$, and moreover that $Y$ is integrable with respect to a certain Young function growing faster than any polynomial. Example 3.1 of the final Section 3 shows that this rate is optimal-possibly up to the value of the constant $\gamma$. The remaining part of Section 2 is devoted to corresponding questions on the growth rate of (higher) derivatives of the flow $\phi$. The main result is Theorem 2.2, which shows that, under differentiability conditions on the local characteristics of $F$, the growth rate of any partial derivative of order at least 1 is at most $Z \exp \left(\gamma(\ln |x|)^{1 / 2}\right)$ for some constant $\gamma>0$, where $Z$ has a similar integrability property as $Y$ above. Example 3.2 of Section 3 proves that the rate is optimal-again, possibly up to the value of the constant $\gamma$.

1. Some estimates of moduli of continuity. In this section we shall provide some estimates of the moduli of continuity of random fields, derived from various assumptions on the moments of their distance at two points of the parameter space. Of course, in later applications of these estimates we shall be interested in quite particular random fields: stochastic flows, where the parameter is just their spatial variable. The assumptions we start with in the following theorems on the moments of distances shall be verified for the flows to be discussed in the following section. The passage from these assumptions to moduli of continuity will be done via the real variable lemma of Garsia, Rodemich and Rumsey. We use the following general version of this lemma in which majorizing measures are crucial (see [1]). For the terminology and the use of majorizing measures, see [6]. 
In the sequel let $(X, d),(M, \rho)$ be separable metric spaces, $m$ a locally finite measure on the Borel sets of $X$. For a function $g: X \rightarrow M$, denote

$$
\tilde{g}(s, t)= \begin{cases}\frac{\rho(g(s), g(t))}{d(s, t)}, & \text { if } s \neq t, \\ 0, & \text { if } s=t,\end{cases}
$$

and let $\Phi:[0, \infty[\rightarrow[0, \infty[$ be an increasing, right-continuous function satisfying $\Phi(0)=0, \Phi(x)>0$ for $x>0$.

Then the extended form of the lemma of Garsia, Rodemich and Rumsey that we shall use can be stated as follows. If $g$ is a continuous function, and

$$
V=\int_{X} \int_{X} \Phi(\tilde{g}(s, t)) m(d s) m(d t)<\infty
$$

then for any $s, t \in X$,

$$
\rho(g(s), g(t)) \leq 12 \max _{z \in\{s, t\}} \int_{0}^{d(s, t)} \Phi^{-1}\left[\frac{4 V}{m\left(K_{\varepsilon}(z)\right)^{2}}\right] d \varepsilon,
$$

where $K_{\varepsilon}(z)$ denotes the closed ball of radius $\varepsilon$ around $z$. For a proof of (2), see [1].

For the rest of this section, let $X=\mathbf{R}^{d}$, fix $\alpha>1$ and choose the majorizing measure $m$ defined by

$$
m(d x)=f(|x|) \lambda(d x),
$$

where $\lambda$ denotes Lebesgue measure on $\mathbf{R}^{d}$ and for $z \geq 0$,

$$
f(z)=\frac{1}{z^{d}\left(\ln ^{+} z\right)^{\alpha} \vee 1} .
$$

We choose this particular density $f$ because it is "just" integrable over $\mathbf{R}^{d}$. The fact that the measure $m$ is finite will be important later on when we establish the finiteness of moments of random variables (such as $Y$ in the following theorem). Let $(\Omega, \mathscr{T}, P)$ be an arbitrary probability space. We emphasize that constants appearing in the inequalities of the paper will be consecutively numbered $c_{1}, c_{2}, c_{3}, \ldots$. The positive part of the logarithm will be written $\ln ^{+} x=\ln x \vee 0$ for $x \geq 0$.

THEOREM 1.1. Let $\phi: \Omega \times \mathbf{R}^{d} \rightarrow M$ be a measurable map such that for any $\omega \in \Omega$ the function $\phi(\omega, \cdot)$ is continuous. Assume that for some $p>2 d$ and some constant $c>0$ we have

$$
E\left(\rho(\phi(\cdot, x), \phi(\cdot, y))^{p}\right) \leq c|x-y|^{p} \quad \text { for } x, y \in \mathbf{R}^{d} .
$$

Then there exists a p-integrable random variable $Y$ such that for all $x, y \in$ $\mathbf{R}^{d}$ such that $|x| \geq|y|$ and all $\omega \in \Omega$, we have

$$
\rho(\phi(\omega, x), \phi(\omega, y)) \leq Y(\omega)|x-y|^{1-2 d / p}\left(\left[|x|^{2 d / p}\left(\ln ^{+}|x|\right)^{2 \alpha / p}\right] \vee 1\right) .
$$

In particular, for all $x \in \mathbf{R}^{d}, \omega \in \Omega$,

$$
\rho(\phi(\omega, x), \phi(\omega, 0)) \leq Y(\omega)\left[\left(|x|\left(\ln ^{+}|x|\right)^{2 \alpha / p}\right) \vee|x|^{1-2 d / p}\right] .
$$


Proof. Set

$$
Z(\omega)=\left[\int_{\mathbf{R}^{d}} \int_{\mathbf{R}^{d}}\left(\frac{\rho(\phi(\omega, x), \phi(\omega, y))}{|x-y|}\right)^{p} m(d x) m(d y)\right]^{1 / p}
$$

$\omega \in \Omega$. Due to the separability of $M, Z$ is measurable. Moreover, (3) implies that $E\left(Z^{p}\right)<\infty$. To apply the GRR lemma, let $\Phi(x)=x^{p}, x \geq 0$. Then (1) yields for $\omega \in \Omega, x, y \in \mathbf{R}^{d}$,

$$
\rho(\phi(\omega, x), \phi(\omega, y)) \leq Z(\omega) c_{1} \max _{z \in\{x, y\}} \int_{0}^{|x-y|} m\left(K_{\varepsilon}(z)\right)^{-2 / p} d \varepsilon .
$$

Assuming $|x| \geq|y|$, we obtain

$$
\begin{aligned}
& \rho(\phi(\omega, x), \phi(\omega, y)) \\
& \quad \leq c_{2} Z(\omega) \int_{0}^{|x-y|} \varepsilon^{-2 d / p} f(|x|+|x-y|)^{-2 / p} d \varepsilon \\
& \quad \leq c_{2} Z(\omega) \frac{|x-y|^{1-2 d / p}}{1-2 d / p}\left[\left((|x|+|x-y|)^{2 d / p}\left(\ln ^{+}(|x|+|x-y|)\right)^{2 \alpha / p}\right) \vee 1\right] \\
& \quad \leq c_{3} Z(\omega)|x-y|^{1-2 d / p}\left[\left(|x|^{2 d / p}\left(\ln ^{+}|x|\right)^{2 \alpha / p}\right) \vee 1\right] .
\end{aligned}
$$

Setting $Y=c_{3} Z$, we get the first assertion of the theorem. The final one is obtained by specializing the first one to $y=0$.

REMARK. Fix $y \in \mathbf{R}^{d}$. Note that condition (3) is translation invariant. We therefore obtain a random variable $Y_{1}$, which is $p$-integrable such that

$$
\rho(\phi(\omega, x), \phi(\omega, y)) \leq Y_{1}(\omega)\left[\left(|x-y|\left(\ln ^{+}|x-y|\right)^{2 \alpha / p}\right) \vee 1\right]
$$

for all $x \in \mathbf{R}^{d}, \omega \in \Omega$. The random variable $Y_{1}$, however, depends on $y$ (or $x$ ).

If (3) holds for all sufficiently large $p$ and if one is able to control the constant $c$ appearing in (3) as a function of $p$, one may, as will be pointed out in the following section, obtain an "integrated" version of (3). In this inequality, the following exponential Young function appears. For $c>0$, let

$$
\Phi_{c}:\left[0, \infty\left[\rightarrow \left[0, \infty\left[, \quad x \mapsto \int_{1}^{\infty} \exp \left(-c t^{2}\right) x^{t} d t .\right.\right.\right.\right.
$$

This function was first introduced in [4] to obtain moduli of continuity for the local time of one-dimensional diffusions in the spatial parameter and used in [2] for establishing conditions under which the multiplicative ergodic theorem holds. The significance of the functions $\Phi_{c}$ is that solutions of SDE's with Lipschitz coefficients have finite $\Phi_{c}$-moments for some (but usually not all) $c>0$. This will be shown in Section 2 .

We need the following estimates of $\Phi_{c}$ and its inverse (see also [2]).

LEMMA 1.1. Let $c>0$, and denote

$$
K=\exp \left[\left(-4 c \ln \left(\int_{1}^{\infty} \exp \left(-c p^{2}\right) d p \wedge 1\right)\right)^{1 / 2}\right] .
$$


Then for $t \geq 0$ we have:

(a) $\Phi_{c}^{-1}(t) \leq K \exp \left(\sqrt{4 c \ln ^{+} t}\right)$;

(b) $\Phi_{c}(t) \leq \sqrt{\pi / c} \exp \left((\ln t)^{2} / 4 c\right)$.

PROOF. A straightforward computation shows that

$$
\exp \left((\ln x)^{2} / 4 c\right) \exp \left(-(\ln K)^{2} / 4 c\right) \leq \Phi_{c}(x) \leq \sqrt{\frac{\pi}{c}} \exp \left((\ln x)^{2} / 4 c\right),
$$

where the left inequality holds for all $x \geq 1$ and the right one for all $x \geq 0$. From this, (a) and (b) follow immediately.

Lemma 1.1 enables us to obtain an "integrated" version of Theorem 1.1.

THEOREM 1.2. Let $\phi: \Omega \times \mathbf{R}^{d} \rightarrow M$ be a measurable map such that for any $\omega \in \Omega$ the function $\phi(\omega, \cdot)$ is continuous. Assume that there exists $c \geq 0$ such that for any $p \geq 1$ we have

$$
E\left(\rho(\phi(\cdot, x), \phi(\cdot, y))^{p}\right) \leq \exp \left(c p^{2}\right)|x-y|^{p}
$$

for all $x, y \in \mathbf{R}^{d}$. Then for any $b>c$ there exists a $\Phi_{b}$-integrable random variable $Y$ such that for all $x, y \in \mathbf{R}^{d}$ such that $|x| \geq|y|$ and all $\omega \in \Omega$,

$$
\begin{aligned}
& \rho(\phi(\omega, x), \phi(\omega, y)) \\
& \leq Y(\omega)(|x| \vee 1) \exp \left(\left[8 b \alpha \ln ^{+} \ln ^{+}|x|\right]^{1 / 2}\right) \\
& \quad \times \int_{0}^{|x-y| /(|x| \vee 1)} \exp \left(\left[8 b d \ln \left(1+\frac{1}{z}\right)\right]^{1 / 2}\right) d z .
\end{aligned}
$$

In particular, there exists a $\Phi_{b}$-integrable random variable $Z$ such that for any $x \in \mathbf{R}^{d}$ and $\omega \in \Omega$,

$$
\rho(\phi(\omega, x), \phi(\omega, 0)) \leq Z(\omega)(|x| \vee 1) \exp \left(\left[8 b \alpha \ln ^{+} \ln ^{+}|x|\right]^{1 / 2}\right) .
$$

REMARK. The finiteness of the integral in (6) is easy to check.

Proof. Let $b>a>c$ and define

$$
U=\Phi_{a}^{-1}\left[\int_{\mathbf{R}^{d}} \int_{\mathbf{R}^{d}} \Phi_{a}\left(\frac{\rho(\phi(\cdot, x), \phi(\cdot, y))}{|x-y|}\right) m(d x) m(d y)\right] .
$$

Since $a>c$, it is easy to see, using the theorem of Fubini, that

$$
E\left(\Phi_{a}(U)\right)<\infty .
$$

Now apply (2) to $\phi$ and $\Phi_{a}$ to obtain the inequality

$$
\rho(\phi(\omega, x), \phi(\omega, y)) \leq c_{4} \max _{z \in\{x, y\}} \int_{0}^{|x-y|} \Phi_{a}^{-1}\left(\frac{c_{5} \Phi_{a}(U)}{m\left(K_{\varepsilon}(z)\right)^{2}}\right) d \varepsilon .
$$


We next use (a) of Lemma 1.1, the inequality $\sqrt{v+w} \leq \sqrt{v}+\sqrt{w}$ for $v, w \geq$ 0 , and the notation

$$
V=\exp \left(\sqrt{4 a \ln ^{+} \Phi_{a}(U)}\right)
$$

For $|x| \geq|y|, \omega \in \Omega$, we then get

$$
\begin{aligned}
\rho(\phi(\omega, x), \phi(\omega, y)) \\
\leq c_{4} K V(\omega) \max _{z \in\{x, y\}} \int_{0}^{|x-y|} \exp \left(\left[4 b\left(\ln ^{+} c_{5}+\ln ^{+}\left(m\left(K_{\varepsilon}(z)\right)^{-2}\right)\right)\right]^{1 / 2}\right) d \varepsilon \\
\leq c_{6} V(\omega) \int_{0}^{|x-y|} \exp \left(\left[4 b \ln ^{+}\left(\varepsilon^{-2 d} f(|x|+\varepsilon)^{-2}\right)\right]^{1 / 2}\right) d \varepsilon \\
\leq c_{7} V(\omega) \exp \left(\left[8 b \alpha \ln ^{+} \ln ^{+}|x|\right]^{1 / 2}\right) \\
\quad \times \int_{0}^{|x-y|} \exp \left(\left[8 b d \ln \left(1+\frac{|x| \vee 1}{\varepsilon}\right)\right]^{1 / 2}\right) d \varepsilon .
\end{aligned}
$$

Furthermore,

$$
\begin{aligned}
\int_{0}^{|x-y|} & \exp \left(\left[8 b d \ln \left(1+\frac{|x| \vee 1}{\varepsilon}\right)\right]^{1 / 2}\right) d \varepsilon \\
= & (|x| \vee 1) \int_{0}^{|x-y| /(|x| \vee 1)} \exp \left(\left[8 b d \ln \left(1+\frac{1}{z}\right)\right]^{1 / 2}\right) d z .
\end{aligned}
$$

Part (b) of Lemma 1.1 guarantees that $V$ is $\Phi_{a}$-integrable and therefore any constant multiple of $V$ is $\Phi_{b}$-integrable. So (9) employed in (8) yields the first assertion, while the second is obtained by setting $y=0$.

We finally consider an application of the real variable lemma to a moment inequality which is slightly, but essentially, different from (5).

THEOREM 1.3. Let $\phi: \Omega \times \mathbf{R}^{d} \rightarrow M$ be a measurable map such that for any $\omega \in \Omega$ the function $\phi(\omega, \cdot)$ is continuous. Assume that there exists $c \geq 0$ such that for any $p \geq 1$ we have

$$
E\left(\rho(\phi(\cdot, x), \phi(\cdot, y))^{p}\right) \leq \exp \left(c p^{2}\right)\left[|x-y|^{p} \wedge 1\right]
$$

for all $x, y \in \mathbf{R}^{d}$. Then for any $b>c$ there exists a $\Phi_{b}$-integrable random variable $Y$ such that for all $x, y \in \mathbf{R}^{d}$ such that $|x| \geq|y|$ and all $\omega \in \Omega$,

$$
\begin{aligned}
& \rho(\phi(\omega, x), \phi(\omega, y)) \\
& \quad \leq Y(\omega) \exp \left(\left[8 b d \ln ^{+}|x|\right]^{1 / 2}\right) \int_{0}^{|x-y| \wedge 1} \exp \left(\left[8 b d \ln ^{+}\left(\frac{1}{\varepsilon}\right)\right]^{1 / 2}\right) d \varepsilon .
\end{aligned}
$$

PROOF. We proceed just as in the preceding proof, except that we now use the metric $d(x, y)=|x-y| \wedge 1$. 
Fix $a$ such that $b>a>c$ and define

$$
Z=\Phi_{a}^{-1}\left(\int_{\mathbf{R}^{d}} \int_{\mathbf{R}^{d}} \Phi_{a}\left(\frac{\rho(\phi(\cdot, x), \phi(\cdot, y))}{d(x, y)}\right) m(d x) m(d y)\right) .
$$

Then (10) implies

$$
E\left(\Phi_{a}(Z)\right)<\infty .
$$

Now we apply (2) with the modified metric, define

$$
V=\exp \left(\left[4 a \ln ^{+}\left(\Phi_{a}(Z)\right)\right]^{1 / 2}\right),
$$

to get for $x, y \in \mathbf{R}^{d}$ such that $|x| \geq|y|$ and $\omega \in \Omega$,

$$
\begin{aligned}
& \rho(\phi(\omega, x), \phi(\omega, y)) \\
& \leq c_{8} V(\omega) \exp \left(\left[8 a \ln ^{+}\left((f(|x|+|x-y|))^{-1}\right)\right]^{1 / 2}\right) \\
& \quad \times \int_{0}^{|x-y| \wedge 1} \exp \left(\left[8 a d \ln ^{+}\left(\frac{1}{\varepsilon}\right)\right]^{1 / 2}\right) d \varepsilon \\
& \leq c_{9} V(\omega) \exp \left(\left[8 b d \ln ^{+}|x|\right]^{1 / 2}\right) \int_{0}^{|x-y| \wedge 1} \exp \left(\left[8 b d \ln ^{+}\left(\frac{1}{\varepsilon}\right)\right]^{1 / 2}\right) d \varepsilon .
\end{aligned}
$$

Another appeal to part (b) of Lemma 1.1 guarantees the $\Phi_{a}$-integrability of $V$ and hence the $\Phi_{b}$-integrability of $c_{9} V$. Hence (11) yields the desired inequality.

2. Spatial estimates for stochastic flows. In this section we shall show that stochastic flows which are generated by stochastic differential equations driven by continuous semimartingale noise satisfy inequalities of the types on which Theorems 1.1, 1.2 and 1.3 were based. Their local characteristics just have to fulfill suitable Lipschitz and growth conditions. The moment inequalities are derived in the following propositions. They lead to our main results, stated in Theorems 2.1 and 2.2, on bounds for the asymptotic spatial growth of the flows and their derivatives.

We will use the set-up and notation of [5] which we recall for the reader's convenience.

Let $F(x, t), t \geq 0$, be a family of $\mathbf{R}^{d}$-valued continuous semimartingales on a filtered probability space $\left(\Omega, \mathscr{F},\left(\mathscr{F}_{t}\right)_{t \geq 0}, P\right)$ indexed by $x \in \mathbf{R}^{d}$. Let $F(x, t)=$ $M(x, t)+V(x, t)$ be the canonical decomposition into a local martingale $M$ and a process $V$ of locally bounded variation. We will assume throughout that both $M$ and $V$ are jointly continuous in $(x, t)$. Furthermore, we assume that there exist $a: \mathbf{R}^{d} \times \mathbf{R}^{d} \times\left[0, \infty\left[\times \Omega \rightarrow \mathbf{R}^{d \times d}\right.\right.$ and $b: \mathbf{R}^{d} \times\left[0, \infty\left[\times \Omega \rightarrow \mathbf{R}^{d}\right.\right.$, called the local characteristics of $F$, such that

$$
\left\langle M_{i}(x, \cdot), M_{j}(y, \cdot)\right\rangle(t)=\int_{0}^{t} a_{i j}(x, y, u) d u, \quad V_{i}(x, t)=\int_{0}^{t} b_{i}(x, u) d u .
$$


Let $\Delta=\left\{(x, x): x \in \mathbf{R}^{d}\right\}$. For $\alpha=\left(\alpha_{1}, \ldots, \alpha_{d}\right), \alpha_{i} \in \mathbf{N}_{0}, i=1, \ldots d$, we write $|\alpha|:=\sum_{i=1}^{d} \alpha_{i}$ as usual.

We shall say that $F$ has local characteristics of class $B_{u b}^{m, \delta}$ for $m \in \mathbf{N}_{0}$, $0<\delta \leq 1$ (or just $F \in B_{u b}^{m, \delta}$ ) if $b$ is in $C^{m}$, and all derivatives of $a$ up to order $m$ with respect to $x$ and $y$ (simultaneously) are continuous and if for all $T>0$,

$$
\operatorname{ess} \sup \sup _{\omega \in \Omega}\left(\left\|a(t) \tilde{\|}_{m+\delta}+\right\| b(t) \|_{m+\delta}\right)<\infty
$$

where

$$
\begin{aligned}
\| a(t) \tilde{\|}_{m+\delta}= & \sup _{x, y \in \mathbf{R}^{d}} \frac{|a(x, y, t)|}{(1+|x|)(1+|y|)}+\sum_{1 \leq|\alpha| \leq m} \sup _{x, y \in \mathbf{R}^{d}}\left|D_{x}^{\alpha} D_{y}^{\alpha} a(x, y, t)\right| \\
& +\sum_{|\alpha|=m} \| D_{x}^{\alpha} D_{y}^{\alpha} a(x, y, t) \tilde{\|}_{\delta}, \\
\|b(t)\|_{m+\delta}= & \sup _{x \in \mathbf{R}^{d}} \frac{|b(x, t)|}{(1+|x|)}+\sum_{1 \leq|\alpha| \leq m} \sup _{x \in \mathbf{R}^{d}}\left|D_{x}^{\alpha} b(x, t)\right| \\
& +\sum_{|\alpha|=m} \sup _{(x, y) \in \Delta^{c}} \frac{\left|D_{x}^{\alpha} b(x, t)-D_{y}^{\alpha} b(y, t)\right|}{|x-y|^{\delta}}, \\
\| f \tilde{\|}_{\delta}:= & \sup \left\{\frac{\left|f(x, y)-f\left(x^{\prime}, y\right)-f\left(x, y^{\prime}\right)+f\left(x^{\prime}, y^{\prime}\right)\right|}{\left|x-x^{\prime}\right|^{\delta}\left|y-y^{\prime}\right|^{\delta}}:\left(x, x^{\prime}\right),\left(y, y^{\prime}\right) \in \Delta^{c}\right\} .
\end{aligned}
$$

Throughout the rest of the paper we will consider the stochastic differential equation

$$
d X(t)=F(X(t), d t)
$$

on $\mathbf{R}^{d}$ where $F$ is a spatial semimartingale as above. If $F \in B_{u b}^{0,1}$ (in fact, even under a slightly weaker condition) Kunita [5], page 155, proved the existence of a stochastic flow of homeomorphisms (or diffeomorphisms, if $F \in B_{u b}^{k, \delta}$ for some $k \geq 1$ ) associated with (12), that is, a map $\phi:\left[0, \infty\left[\times\left[0, \infty\left[\times \mathbf{R}^{d} \times \Omega \rightarrow \mathbf{R}^{d}\right.\right.\right.\right.$ such that:

1. $\phi_{s t}(x, \cdot), t \geq s$ solves (12) with initial condition $X(s)=x$ for each $s \geq 0$, $x \in \mathbf{R}^{d}$;

2. $\phi_{s t}(\cdot, \omega)$ is a homeomorphism for each $0 \leq s \leq t, \omega \in \Omega$;

3. $\phi_{s t}(\cdot, \omega)=\phi_{t s}^{-1}(\cdot, \omega)$ for each $s, t \geq 0, \omega \in \Omega$;

4. $\phi_{s u}(\cdot, \omega)=\phi_{t u}(\cdot, \omega) \circ \phi_{s t}(\cdot, \omega)$ for all $s, t, u \geq 0, \omega \in \Omega$;

5. $(s, t) \mapsto \phi_{s t}(\cdot, \omega)$ is continuous from $[0, \infty)^{2}$ to the (group of) homeomorphisms on $\mathbf{R}^{d}$.

In the following, $\phi$ will always denote the flow associated with (12). We remark that all moments of the flow and its derivatives appearing in the sequel are finite, according to [5]. 
Proposition 2.1. Assume $F \in B_{u b}^{0,1}$. Fix $T>0$. Then there exists $c \geq 0$ such that for all $x, y \in \mathbf{R}^{d}$ and all $p \geq 1$, we have:

(a) $E \sup _{0 \leq t \leq T}\left(\left|\phi_{0 t}(x)-\phi_{0 t}(y)\right|^{p}\right) \leq \exp \left(c p^{2}\right)|x-y|^{p}$;

(b) $E \sup _{0 \leq t \leq T}\left(\left|\phi_{0 t}(0)\right|^{p}\right) \leq \exp \left(c p^{2}\right)$.

Proof. It is enough to prove (a) and (b) for $p \geq 4$.

(a) Since $F \in B_{u b}^{0,1}$ there exists $c_{10} \geq 0$ such that for all $x, \tilde{x}, y, \tilde{y} \in \mathbf{R}^{d}$, $0 \leq t \leq T$ and $\omega \in \Omega$,

$$
|a(x, y, t)-a(\tilde{x}, y, t)-a(x, \tilde{y}, t)+a(\tilde{x}, \tilde{y}, t)| \leq c_{10}|x-\tilde{x}||y-\tilde{y}|
$$

and

$$
|b(x, t)-b(y, t)| \leq c_{10}|x-y| .
$$

Fix $x, y \in \mathbf{R}^{d}$ and define

$$
Y(t):=\left|\phi_{0 t}(x)-\phi_{0 t}(y)\right|^{2}=\sum_{i=1}^{d}\left(\phi_{0 t}(x)-\phi_{0 t}(y)\right)_{i}^{2} .
$$

Then Itô's formula implies

$$
\begin{gathered}
d Y(t)=\sum_{i=1}^{d} 2\left(\phi_{0 t}(x)-\phi_{0 t}(y)\right)_{i}\left(M_{i}\left(\phi_{0 t}(x), d t\right)-M_{i}\left(\phi_{0 t}(y), d t\right)\right) \\
\quad+\sum_{i=1}^{d} 2\left(\phi_{0 t}(x)-\phi_{0 t}(y)\right)_{i}\left(b_{i}\left(\phi_{0 t}(x), t\right)-b_{i}\left(\phi_{0 t}(y), t\right)\right) d t \\
+\sum_{i=1}^{d} d\left\langle M_{i}\left(\phi_{0 t}(x), \cdot\right)-M_{i}\left(\phi_{0 t}(y), \cdot\right)\right\rangle_{t} .
\end{gathered}
$$

Therefore we obtain for $p \geq 2$ and $t \leq T$,

$$
\begin{aligned}
& E\left(\sup _{0 \leq s \leq t} Y(s)^{p}\right) \\
& \leq 3^{p-1}\left(|x-y|^{2 p}+E \sup _{0 \leq s \leq t} \mid \sum_{i=1}^{d} 2 \int_{0}^{s}\left(\phi_{0 u}(x)-\phi_{0 u}(y)\right)_{i}\right. \\
& \quad \times\left.\left(M_{i}\left(\phi_{0 u}(x), d u\right)-M_{i}\left(\phi_{0 u}(y), d u\right)\right)\right|^{p} \\
& +E\left(\int_{0}^{t} 2 c_{10}\left|\phi_{0 u}(x)-\phi_{0 u}(y)\right|^{2}\right. \\
& \quad+\sum_{i=1}^{d} \mid a_{i i}\left(\phi_{0 u}(x), \phi_{0 u}(x), u\right)-a_{i i}\left(\phi_{0 u}(x), \phi_{0 u}(y), u\right) \\
& \left.\left.\quad-a_{i i}\left(\phi_{0 u}(y), \phi_{0 u}(x), u\right)+a_{i i}\left(\phi_{0 u}(y), \phi_{0 u}(y), u\right) \mid d u\right)^{p}\right)
\end{aligned}
$$




$$
\begin{aligned}
& \leq 3^{p-1}\left(|x-y|^{2 p}+C(p) E\left(\int_{0}^{t} 4 c_{10}\left|\phi_{0 u}(x)-\phi_{0 u}(y)\right|^{4} d u\right)^{p / 2}\right. \\
& \left.+E\left(\int_{0}^{t}(2+d) c_{10}\left|\phi_{0 u}(x)-\phi_{0 u}(y)\right|^{2} d u\right)^{p}\right),
\end{aligned}
$$

where $C(p)=\left(c_{11} p^{1 / 2}\right)^{p}$ is an upper bound for the constant in Burkholder's inequality for continuous martingales for all $p \geq 2$, and $c_{11}$ is some universal constant ([3], page 207).

Abbreviating $f(t):=\left(E \sup _{0 \leq s \leq t} Y(s)^{p}\right)^{1 / p}$ we get

$$
f(t) \leq 3\left(|x-y|^{2}+C(p)^{1 / p} 2 \sqrt{c_{10}}\left(\int_{0}^{t} f^{2}(u) d u\right)^{1 / 2}+c_{10}(2+d) \int_{0}^{t} f(u) d u\right)
$$

and hence

$$
f^{2}(t) \leq 27\left(|x-y|^{4}+C(p)^{2 / p} 4 c_{10} \int_{0}^{t} f^{2}(u) d u+c_{10}^{2}(2+d)^{2} T \int_{0}^{t} f^{2}(u) d u\right) .
$$

Using Gronwall's inequality, we obtain

$$
\begin{aligned}
& E \sup _{0 \leq t \leq T}\left|\phi_{0 t}(x)-\phi_{0 t}(y)\right|^{2 p} \\
& \quad \leq|x-y|^{2 p} 27^{p / 2} \exp \left(\frac{27 p T}{2}\left(C(p)^{2 / p} 4 c_{10}+c_{10}^{2}(2+d)^{2} T\right)\right) .
\end{aligned}
$$

Since $C(p)^{1 / p}=c_{11} p^{1 / 2}$, the assertion follows.

(b) Let $Y(t)=\left|\phi_{0 t}(0)\right|^{2}$. Then

$$
\begin{aligned}
d Y(t)= & \sum_{i=1}^{d} 2 \phi_{0 t}(0)_{i} M_{i}\left(\phi_{0 t}(0), d t\right)+\sum_{i=1}^{d} 2 \phi_{0 t}(0)_{i} b_{i}\left(\phi_{0 t}(0), t\right) d t \\
& +\sum_{i=1}^{d} d\left\langle M_{i}\left(\phi_{0 t}(0), t\right)\right\rangle .
\end{aligned}
$$

Using the fact that there exists $c_{12} \geq 0$ such that

$$
|a(x, x, t)| \leq c_{12}\left(1+|x|^{2}\right)
$$

and

$$
|b(x, t)| \leq c_{12}(1+|x|)
$$

for all $x \in \mathbf{R}^{d}, 0 \leq t \leq T$, and $\omega \in \Omega$, the assertion follows as in part (a).

REMARK. The good estimate $C(p)=\left(c_{11} p^{1 / 2}\right)^{p}$ of the best constant in Burkholder's inequality, which showed up in the preceding proof, is crucial for our estimates and will appear in subsequent proofs repeatedly.

Proposition 2.1 combined with Theorem 1.2 provide spatial estimates for $\sup _{0 \leq t \leq T}\left|\phi_{0 t}(x)\right|$. Together with the following proposition we shall be able to extend this estimate even to $\sup _{0 \leq s, t \leq T}\left|\phi_{s t}(x)\right|$ in Theorem 2.1. 
Proposition 2.2. Assume $F \in B_{u b}^{0,1}$ and define

$$
\Psi_{t}(x):=\frac{|x|^{2}}{1+\left|\phi_{0 t}(x)\right|}, \quad t \geq 0, x \in \mathbf{R}^{d} .
$$

For every $T>0$ there exists $c \geq 0$ such that for all $p \geq 1$ and $x, y \in \mathbf{R}^{d}$ :

(a) $E \sup _{0 \leq t \leq T}\left(1+\left|\phi_{0 t}(x)\right|^{2}\right)^{-p} \leq \exp \left(c p^{2}\right)\left(1+|x|^{2}\right)^{-p}$;

(b) $E \sup _{0 \leq t \leq T}\left(\left|\Psi_{t}(x)-\Psi_{t}(y)\right|^{p}\right) \leq \exp \left(c p^{2}\right)|x-y|^{p}$.

Proof. It suffices to prove the assertions for $p \geq 2$.

Fix $x \in \mathbf{R}^{d}, T>0$ and define

$$
X(t):=\phi_{0 t}(x), \quad Y(t):=\left(1+X(t)^{2}\right)^{-1} .
$$

Itô's formula implies

$$
\begin{aligned}
d Y(t)= & -\sum_{i=1}^{d} \frac{2 X_{i}(t)}{\left(1+|X(t)|^{2}\right)^{2}} M_{i}(X(t), d t) \\
& -\sum_{i=1}^{d} \frac{2 X_{i}(t)}{\left(1+|X(t)|^{2}\right)^{2}} b_{i}(X(t), t) d t \\
& -\frac{1}{2} \sum_{i=1}^{d} \frac{2}{\left(1+|X(t)|^{2}\right)^{2}} a_{i i}(X(t), X(t), t) d t \\
& +\frac{1}{2} \sum_{i, j=1}^{d} \frac{8 X_{i}(t) X_{j}(t)}{\left(1+|X(t)|^{2}\right)^{3}} a_{i j}(X(t), X(t), t) d t
\end{aligned}
$$

Since $F \in B_{u b}^{0,1}$, there exists $c_{13} \geq 0$ such that

$$
|a(x, x, t)| \leq c_{13}\left(1+|x|^{2}\right) \text { and }|b(x, t)|^{2} \leq c_{13}^{2}\left(1+|x|^{2}\right) .
$$

Proceeding as in the proof of Proposition 2.1, and denoting

$$
f(t):=\left(E\left(\sup _{0 \leq s \leq t} Y(s)^{p}\right)\right)^{1 / p}, \quad 0 \leq t \leq T, \quad p \geq 2,
$$

we obtain

$$
\begin{array}{r}
f(t) \leq 3\left[\left(1+|x|^{2}\right)^{-1}+C(p)^{1 / p} 2 \sqrt{c_{13}}\left(\int_{0}^{t} f(s)^{2} d s\right)^{1 / 2}\right. \\
\left.+(2+d+4) c_{13} \int_{0}^{t} f(s) d s\right] .
\end{array}
$$

The assertion now follows by applying Gronwall's lemma to $f^{2}$ as in the proof of Proposition 2.1. 
To prove (b), note that for $x, y \in \mathbf{R}^{d},|y| \geq|x|$, and $0 \leq t \leq T$, we have

$$
\begin{aligned}
& \left|\Psi_{t}(x)-\Psi_{t}(y)\right| \\
& \quad \leq|x|^{2}\left|\frac{1}{1+\left|\phi_{0 t}(x)\right|}-\frac{1}{1+\left|\phi_{0 t}(y)\right|}\right|+\left.\frac{1}{1+\left|\phi_{0 t}(y)\right|}|| x\right|^{2}-|y|^{2} \mid \\
& \quad \leq \frac{|x|}{1+\left|\phi_{0 t}(x)\right|} \frac{|y|}{1+\left|\phi_{0 t}(y)\right|}\left|\phi_{0 t}(x)-\phi_{0 t}(y)\right|+|x-y| \frac{2|y|}{1+\left|\phi_{0 t}(y)\right|} .
\end{aligned}
$$

Now the assertion follows from Proposition 2.1 and part (a).

We are ready to state and prove our main result on asymptotic growth rates for stochastic flows.

TheOREM 2.1. Assume $F \in B_{u b}^{0,1}$. Then for all $T>0$, there exist $c, \gamma>0$ such that

$$
Y:=\sup _{x \in \mathbf{R}^{d}} \frac{1+\sup _{0 \leq s, t \leq T}\left|\phi_{s t}(x)\right|}{|x|+1} \exp \left(-\gamma\left(\ln ^{+} \ln ^{+}|x|\right)^{1 / 2}\right)
$$

and

$$
Y^{\prime}:=\sup _{x \in \mathbf{R}^{d}} \sup _{0 \leq s, t \leq T} \frac{|x|+1}{1+\left|\phi_{s t}(x)\right|} \exp \left(-\gamma\left(\ln ^{+} \ln ^{+}|x|\right)^{1 / 2}\right)
$$

are $\Phi_{c}$-integrable.

Proof. Fix $x \in \mathbf{R}^{d}$. For $0 \leq s \leq T$, define $y_{s}:=\phi_{0 s}^{-1}(x, \cdot)$. Propositions 2.1 and 2.2 together with Theorem 1.2 show that there exist $c_{14}, c_{15}>0$ and a $\Phi_{c_{14}}$-integrable random variable $Z$ (not depending on $x$ ), such that for all $0 \leq t \leq T$,

$$
\begin{aligned}
& 1+\left|\phi_{0 t}\left(y_{s}\right)\right| \leq Z\left(\left|y_{s}\right|+1\right) \exp \left(c_{15}\left(\ln ^{+} \ln ^{+}\left|y_{s}\right|\right)^{1 / 2}\right), \\
& 1+\left|\phi_{0 t}\left(y_{s}\right)\right| \geq Z^{-1}\left(\left|y_{s}\right|+1\right) \exp \left(-c_{15}\left(\ln ^{+} \ln ^{+}\left|y_{s}\right|\right)^{1 / 2}\right) .
\end{aligned}
$$

To obtain (13), first apply (b) in Proposition 2.1 to get the $\Phi_{c_{14}}$-integrability of $\sup _{0 \leq t \leq T}\left|\phi_{0 t}(0)\right|$, then use (a). Then (13) and (14) imply

$$
1+\left|\phi_{s t}(x)\right|=1+\left|\phi_{0 t}\left(y_{s}\right)\right| \leq Z^{2}(1+|x|) \exp \left(2 c_{15}\left(\ln ^{+} \ln ^{+}\left|y_{s}\right|\right)^{1 / 2}\right) .
$$

Now (14) implies that there exists $\alpha \geq 0$ (depending on $c_{15}$ ) such that $\left|y_{s}\right|+1 \leq$ $\alpha^{2} Z^{2}(1+|x|)^{2}$, so, assuming w.l.o.g. $\ln ^{+}\left(\alpha^{2} Z^{2}\right) \geq 1$,

$$
\begin{aligned}
\left(\ln ^{+} \ln ^{+}\left|y_{s}\right|\right)^{1 / 2} & \leq\left(\ln \left(\ln \left(\alpha^{2} Z^{2}\right)+\ln \left(3(1+|x|)^{2}\right)\right)\right)^{1 / 2} \\
& \leq\left(\ln \ln \left(\alpha^{2} Z^{2}\right)+\ln \ln \left(3(1+|x|)^{2}\right)+\ln 2\right)^{1 / 2} \\
& \leq\left(\ln \ln \left(\alpha^{2} Z^{2}\right)\right)^{1 / 2}+\left(\ln \ln \left(3(1+|x|)^{2}\right)\right)^{1 / 2}+(\ln 2)^{1 / 2}
\end{aligned}
$$


Therefore,

$$
\begin{aligned}
1+\sup _{0 \leq s, t \leq T}\left|\phi_{s t}(x)\right| \leq & Z^{2} \exp \left(2 c_{15}\left(\ln \ln \left(\alpha^{2} Z^{2}\right)\right)^{1 / 2}\right) \exp \left(2 c_{15}(\ln 2)^{1 / 2}\right)(1+|x|) \\
& \times \exp \left(2 c_{15}\left(\ln \ln 3(1+|x|)^{2}\right)^{1 / 2}\right) .
\end{aligned}
$$

Now the first assertion follows with $\gamma=2 c_{15}$.

Further,

$$
1+\left|\phi_{s t}(x)\right|=1+\left|\phi_{0 t}\left(y_{s}\right)\right| \geq Z^{-2}(1+|x|) \exp \left(-2 c_{15}\left(\ln ^{+} \ln ^{+}\left|y_{s}\right|\right)^{1 / 2}\right) .
$$

Using (15), the second assertion follows with $\gamma=2 c_{15}$.

Remarks. (1) Suppose we use the moment inequalities of Propositions 2.1 and 2.2 just for one individual $p>2 d$ instead of the "integrated version" hidden behind the appearance of $\Phi_{c}$ employed in Theorem 2.1. Then, as Theorem 1.1 shows, our analysis just leads to $|x|(\ln |x|)^{\varepsilon}$ for arbitrary $\varepsilon>0$ as estimates of growth rates, which is the bound derived in [10]. This is another confirmation of the power of methods involving the real variable lemma of GRR.

(2) It will be shown in Section 3 that the growth rates appearing in Theorem 2.1 are sharp. This result is also nearly optimal in another respect. The random variable $Y$ is proved to be $\Phi_{c}$-integrable for some $c>0$. As the example of the flow $\phi$ in dimension 1 associated with the SDE

$$
d x_{t}=x_{t} d W_{t}
$$

shows, this integrability statement cannot be improved by much. For fixed $t>0$ and $x \neq 0$, the random variable $\phi_{0 t}(x)$ is not $\Phi_{c}$-integrable for $c$ small enough. This is a fortiori true for $Y$.

We next consider spatial estimates of derivatives of a stochastic flow.

Proposition 2.3. Assume that $F \in B_{u b}^{k, 1}$ for some $k \in \mathbf{N}$. Then for all $T>0$ there exists $c \geq 0$ such that for all $1 \leq|\alpha| \leq k, p \geq 1$ and $x, y \in \mathbf{R}^{d}$ we have

$$
E\left(\sup _{0 \leq t \leq T}\left|D^{\alpha} \phi_{0 t}(x)-D^{\alpha} \phi_{0 t}(y)\right|^{p}\right) \leq \exp \left(c p^{2}\right)\left(|x-y|^{p} \wedge 1\right)
$$

and

$$
E\left(\sup _{0 \leq t \leq T}\left|D^{\alpha} \phi_{0 t}(x)\right|^{p}\right) \leq \exp \left(c p^{2}\right)
$$

Proof. Theorem 4.6.5 in [5] shows that $\phi$ is a flow of $C^{k}$-diffeomorphisms. We denote by $D \phi_{0 t}(x)$ the Jacobian of $\phi_{0 t}$ with respect to $x$ and similarly for $F$. Abusing notation, we shall often write $D^{i}$ for the derivative w.r.t. the 
$i$ th variable. We shall use upper indices to denote the components of $\phi$. For $1 \leq|\alpha| \leq k$ and $j \in\{1, \ldots, d\}$ we have the equation

$$
\begin{aligned}
D^{\alpha} \phi_{0 t}^{j}(x)= & \eta_{\alpha, j}+\sum_{n=1}^{d} \int_{0}^{t} D^{\alpha} \phi_{0 s}^{n}(x) D^{n} F_{j}\left(\phi_{0 s}(x), d s\right) \\
& +\sum_{2 \leq|\beta| \leq|\alpha|} \int_{0}^{t} P_{\beta, x, j}(s) D^{\beta} F_{j}\left(\phi_{0 s}(x), d s\right),
\end{aligned}
$$

where $\eta_{\alpha, j}=1$ if $|\alpha|=1$ and $\alpha_{j}=1$ and $\eta_{\alpha, j}=0$ otherwise, and where $P_{\beta, x, j}(s)$ is a finite sum of products of the form $\prod_{i=1}^{r} D^{\gamma_{i}} \phi_{0 s}^{j_{i}}(x)$ with $1 \leq\left|\gamma_{i}\right|<$ $|\alpha|$ and $\sum_{i=1}^{r}\left|\gamma_{i}\right|=|\alpha|$ (see [5], page 95). Observe that the second sum in (18) is zero in case $|\alpha|=1$.

We first prove (17) by induction on $|\alpha|$. For $1 \leq j \leq d, 0 \leq t \leq T, p \geq 1$ let

$$
\begin{aligned}
& Y_{j}(t)=D^{\alpha} \phi_{0 t}^{j}(x), \quad Y(t)=\left|D^{\alpha} \phi_{0 t}(x)\right|=\left[\sum_{j=1}^{d} Y_{j}^{2}(t)\right]^{1 / 2}, \\
& Z_{j}(t)=\sup _{0 \leq s \leq t}\left|Y_{j}(s)\right|, \quad Z(t)=\sup _{0 \leq s \leq t} Y(s), \\
& f_{p}(t)=\left[E\left(Z(t)^{p}\right)\right]^{1 / p},
\end{aligned}
$$

where we suppress $x$ and $\alpha$ for ease of notation. We rewrite (18) to get

$$
\begin{aligned}
Y_{j}(t)= & \eta_{\alpha, j}+\sum_{n=1}^{d} \int_{0}^{t} Y_{n}(s) D^{n} F_{j}\left(\phi_{0 s}(x), d s\right) \\
& +\sum_{2 \leq|\beta| \leq|\alpha|} \int_{0}^{t} P_{\beta, x, j}(s) D^{\beta} F_{j}\left(\phi_{0 s}(x), d s\right) .
\end{aligned}
$$

With the help of [5], Theorem 3.1.2, which allows us to interchange spatial derivatives and the quadratic variation, we get for $p \geq 2$,

$$
\begin{aligned}
& {\left[E\left(Z_{j}(t)^{p}\right)\right]^{1 / p} \leq c_{16}(1}+C(p)^{1 / p}\left[E\left(\int_{0}^{t} Z(s)^{2} d s\right)^{p / 2}\right]^{1 / p}+\int_{0}^{t} f_{p}(s) d s \\
&+C(p)^{1 / p} \sum_{j, \beta}\left[E\left(\int_{0}^{t} P_{\beta, x, j}^{2}(s) d s\right)^{p / 2}\right]^{1 / p} \\
&\left.+\sum_{j, \beta} \int_{0}^{t}\left[E\left(\left|P_{\beta, x, j}(s)\right|^{p}\right)\right]^{1 / p} d s\right) .
\end{aligned}
$$

Therefore, for $0 \leq t \leq T$ we obtain the estimate

$$
\begin{aligned}
f_{p}^{2}(t) \leq c_{17}\left(\left(C(p)^{2 / p}+1\right) \int_{0}^{t}\right. & f_{p}^{2}(s) d s+\left(C(p)^{2 / p}+1\right) \\
& \left.\times \sum_{j, \beta} \int_{0}^{t}\left[E\left(\left|P_{\beta, x, j}(s)\right|^{p}\right)\right]^{2 / p} d s\right) .
\end{aligned}
$$


For $|\alpha|=1$ the last sum is empty and consequently we get (17). For $1<|\alpha| \leq k$ we use the induction hypothesis and Hölder's inequality to show that for some constant $c_{18} \geq 0$ and all $p \geq 1$ we have

$$
E\left(\left|P_{\beta, x, j}(s)\right|^{p}\right) \leq \exp \left(c_{18} p^{2}\right) .
$$

Now Gronwall's lemma applied to $f_{p}^{2}$ implies (17).

It remains to prove (16). Due to (17) it is enough to prove the inequality with $|x-y|^{p} \wedge 1$ replaced by $|x-y|^{p}$. Let $1 \leq|\alpha| \leq k, x, y \in \mathbf{R}^{d}, 1 \leq j \leq d$, $p \geq 1$, and, again suppressing $\alpha, x, y$, define

$$
\begin{aligned}
& V_{j}(t)=D^{\alpha} \phi_{0 t}^{j}(x)-D^{\alpha} \phi_{0 t}^{j}(y), \quad V(t)=\left[\sum_{j=1}^{d} V_{j}^{2}(t)\right]^{1 / 2}, \\
& W_{j}(t)=\sup _{0 \leq s \leq t}\left|V_{j}(s)\right|, \quad W(t)=\sup _{0 \leq s \leq t} V(s), \\
& g_{p}(t)=\left[E\left(W(t)^{p}\right)\right]^{1 / p} .
\end{aligned}
$$

Now we have

$$
\begin{aligned}
& V_{j}(t)=\sum_{n=1}^{d}\left[\int_{0}^{t} D^{\alpha} \phi_{0 s}^{n}(x) D^{n} F_{j}\left(\phi_{0 s}(x), d s\right)-\int_{0}^{t} D^{\alpha} \phi_{0 s}^{n}(y) D^{n} F_{j}\left(\phi_{0 s}(y), d s\right)\right] \\
& +\sum_{2 \leq|\beta| \leq|\alpha|}\left[\int_{0}^{t} P_{\beta, x, j}(s) D^{\beta} F_{j}\left(\phi_{0 s}(x), d s\right)\right. \\
& \left.\quad-\int_{0}^{t} P_{\beta, y, j}(s) D^{\beta} F_{j}\left(\phi_{0 s}(y), d s\right)\right] \\
& =\sum_{n=1}^{d}\left[\int_{0}^{t} V_{j}(s) D^{n} F_{j}\left(\phi_{0 s}(x), d s\right)\right. \\
& \left.\quad+\int_{0}^{t} D^{\alpha} \phi_{0 s}^{n}(y)\left(D^{n} F_{j}\left(\phi_{0 s}(x), d s\right)-D^{n} F_{j}\left(\phi_{0 s}(y), d s\right)\right)\right] \\
& +\sum_{2 \leq|\beta| \leq|\alpha|}\left[\int_{0}^{t}\left(P_{\beta, x, j}(s)-P_{\beta, y, j}(s)\right) D^{\beta} F_{j}\left(\phi_{0 s}(x), d s\right)\right. \\
& \left.\quad+\int_{0}^{t} P_{\beta, y, j}(s)\left(D^{\beta} F_{j}\left(\phi_{0 s}(x), d s\right)-D^{\beta} F_{j}\left(\phi_{0 s}(y), d s\right)\right)\right] .
\end{aligned}
$$

Again, the proof goes by induction on $|\alpha|$. For $|\alpha|=1$, the last sum drops out, and we get for $0 \leq t \leq T$, and $p \geq 2$,

$$
\begin{aligned}
g_{p}^{2}(t) \leq c_{19}\left(\left(C(p)^{2 / p}\right.\right. & +1) \int_{0}^{t} g_{p}^{2}(s) d s+\left(C(p)^{2 / p}+1\right) \\
& \left.\times \int_{0}^{t}\left[E\left(\left|D^{\alpha} \phi_{0 s}(y)\right|^{2 p}\right)\right]^{1 / p}\left[E\left(\left|\phi_{0 s}(x)-\phi_{0 s}(y)\right|^{2 p}\right)\right]^{1 / p} d s\right) .
\end{aligned}
$$


Using (17), Proposition 2.1 and applying Gronwall's lemma we get (16) for $|\alpha|=1$.

For general $2 \leq|\alpha| \leq k$, observe that $P_{\beta, x, j}(s)-P_{\beta, y, j}(s)$ can be expressed as a finite sum of terms of the form $\left[D^{\gamma} \phi_{0 s}^{k}(x)-D^{\gamma} \phi_{0 s}^{k}(y)\right] \prod_{i=1}^{r} D^{\gamma_{i}} \phi_{0 s}^{k_{i}}(z)$ where $z \in\{x, y\}, 1 \leq|\gamma|<\alpha,\left|\gamma_{i}\right| \geq 1$, and $\gamma+\sum_{i=1}^{r}\left|\gamma_{i}\right|=|\alpha|$. Using this fact the induction proof follows along the familiar lines.

We next extend the considerations of Proposition 2.3 to the inverse of the Jacobian of a stochastic flow.

Proposition 2.4. Assume that $F \in B_{u b}^{1,1}$. Let $\mathscr{I}_{s t}(x)=\left[D \phi_{s t}(x)\right]^{-1}, 0 \leq$ $s \leq t$. Then for all $T>0$ there exists $c \geq 0$ such that for all $p \geq 1$ and $x, y \in \mathbf{R}^{d}$, we have

$$
E\left(\sup _{0 \leq t \leq T}\left|\mathscr{I}_{0 t}(x)-\mathscr{I}_{0 t}(y)\right|^{p}\right) \leq \exp \left(c p^{2}\right)\left(|x-y|^{p} \wedge 1\right)
$$

and

$$
E\left(\sup _{0 \leq t \leq T}\left|\mathscr{I}_{0 t}(x)\right|^{p}\right) \leq \exp \left(c p^{2}\right)
$$

PROOF. The following well-known identity is easily checked with Itô's formula:

$$
\mathscr{I}_{0 t}(x)=I-\int_{0}^{t} \mathscr{I}_{0 s}(x) D F\left(\phi_{0 s}(x), d s\right)+\int_{0}^{t} \mathscr{I}_{0 s}(x) \tilde{a}\left(\phi_{0 s}(x), \phi_{0 s}(x), s\right) d s,
$$

where $\tilde{a}^{i j}(x, y, s)=\sum_{k=1}^{d} D_{x}^{k} D_{y}^{j} a_{i k}(x, y, s), i, j \in\{1, \ldots, d\}$. Using the fact that $\tilde{a}$ is bounded, the arguments of the preceding propositions easily yield (21), taking the $p$ th moment of the supremum of $\left|\mathscr{I}_{0 s}^{i j}(x)\right|$ in $s$ between 0 and $t$.

To show (20), we write

$$
\begin{aligned}
\mathscr{I}_{0 t}(x)-\mathscr{I}_{0 t}(y)= & -\int_{0}^{t}\left(\mathscr{I}_{0 s}(x)-\mathscr{I}_{0 s}(y)\right) D F\left(\phi_{0 s}(x), d s\right) \\
& +\int_{0}^{t} \mathscr{I}_{0 s}(y)\left(D F\left(\phi_{0 s}(y), d s\right)-D F\left(\phi_{0 s}(x), d s\right)\right) \\
& +\int_{0}^{t}\left(\mathscr{I}_{0 s}(x)-\mathscr{I}_{0 s}(y)\right) \tilde{a}\left(\phi_{0 s}(x), \phi_{0 s}(x), s\right) d s \\
& +\int_{0}^{t} \mathscr{I}_{0 s}(y)\left(\tilde{a}\left(\phi_{0 s}(x), \phi_{0 s}(x), s\right)\right. \\
& \left.-\tilde{a}\left(\phi_{0 s}(y), \phi_{0 s}(y), s\right)\right) d s .
\end{aligned}
$$

We will be able to show (20) by the arguments of the second part of the proof of Proposition 2.3 for $|\alpha|=1$, once we know that for $\bar{a}^{i j k l}(x, y, t)=$ $D_{x}^{i} D_{y}^{j} a_{k l}(x, y, t), i, j, k, l \in\{1, \ldots, d\}$, we have

$$
\left|\bar{a}^{i j k l}(x, x, t)-\bar{a}^{i j k l}(y, y, t)\right| \leq c_{20}|x-y|
$$


for all $0 \leq t \leq T, x, y \in \mathbf{R}^{d}$, with some constant $c_{20}$. Since $F \in B_{u b}^{1,1}$, we know that there exists a constant $c_{21}$ such that for all $i, j$ and all $x, y \in \mathbf{R}^{d}$, we have

$$
\left|\bar{a}^{i j i j}(x, x, t)-2 \bar{a}^{i j i j}(x, y, t)+\bar{a}^{i j i j}(y, y, t)\right| \leq c_{21}|x-y|^{2}
$$

and

$$
\left|\bar{a}^{i j i j}(x, x, t)\right| \leq c_{21} .
$$

Fix $i, j, k, l$ and write $\bar{M}(x, t)=D^{i} M_{k}(x, t), \bar{N}(x, t)=D^{j} M_{l}(x, t)$. Then, the inequality of Kunita-Watanabe yields

$$
\begin{aligned}
&\left|\bar{a}^{i j k l}(x, x, t)-\bar{a}^{i j k l}(y, y, t)\right| \\
&=\mid \frac{d}{d t}\left(\left\langle\bar{M}(x, \cdot),\left.\bar{N}(x, \cdot)\right|_{t}-\left\langle\bar{M}(y, \cdot),\left.\bar{N}(y, \cdot)\right|_{t}\right)\right|\right. \\
& \leq \mid \frac{d}{d t}\left\langle\bar{M}(x, \cdot)-\bar{M}(y, \cdot),\left.\bar{N}(x, \cdot)\right|_{t}\right| \\
&+\left|\frac{d}{d t}\langle\bar{M}(y, \cdot), \bar{N}(x, \cdot)-\bar{N}(y, \cdot)\rangle_{t}\right| \\
& \leq \sqrt{\frac{d}{d t}\left\langle\bar{M}(x, \cdot)-\left.\bar{M}(y, \cdot)\right|_{t}\right.} \sqrt{\frac{d}{d t}\langle\bar{N}(x, \cdot)\rangle_{t}} \\
&+\sqrt{\frac{d}{d t}\langle\bar{M}(y, \cdot)\rangle_{t}} \sqrt{\frac{d}{d t}\langle\bar{N}(x, \cdot)-\bar{N}(y, \cdot)\rangle_{t}} \\
& \leq 2 c_{21}|x-y| .
\end{aligned}
$$

The estimates of the preceding propositions combined with the results of the first section lead to the following main theorem about the asymptotic growth of the derivatives of a stochastic flow.

TheOREM 2.2. Assume $F \in B_{u b}^{k, 1}$ for some $k \in \mathbf{N}$. Then for all $T>0$, there exist $c, \gamma>0$ such that for all $1 \leq|\alpha| \leq k$ the random variables

$$
\begin{aligned}
Y & =\sup _{x \in \mathbf{R}^{d}} \sup _{0 \leq s, t \leq T}\left|\left(D \phi_{s t}(x)\right)^{-1}\right| \exp \left(-\gamma\left(\ln ^{+}|x|\right)^{1 / 2}\right), \\
Y^{\prime} & =\sup _{x \in \mathbf{R}^{d}} \sup _{0 \leq s, t \leq T} \frac{1}{\left|D \phi_{s t}(x)\right|} \exp \left(-\gamma\left(\ln ^{+}|x|\right)^{1 / 2}\right)
\end{aligned}
$$

and

$$
Y_{\alpha}=\sup _{x \in \mathbf{R}^{d}} \sup _{0 \leq s, t \leq T}\left|D^{\alpha} \phi_{s t}(x)\right| \exp \left(-\gamma\left(\ln ^{+}|x|\right)^{1 / 2}\right)
$$

are $\Phi_{c}$-integrable.

PROOF. Here (23) is an easy consequence of (22) since for any matrix norm submultiplicativity gives $|A|\left|A^{-1}\right| \geq|I|$ for matrices $A$. 
Let us first show (22). Fix $T>0$ and $x \in \mathbf{R}^{d}$. For $0 \leq s \leq T$, define $y_{s}=\phi_{0 s}^{-1}(x)$ as in the proof of Theorem 2.1. The equation

$$
D \phi_{0 t}\left(y_{s}\right)=D \phi_{s t}(x) D \phi_{0 s}\left(y_{s}\right)
$$

implies the estimate

$$
\left|\left(D \phi_{s t}(x)\right)^{-1}\right| \leq\left|D \phi_{0 s}\left(y_{s}\right)\right|\left|D \phi_{0 t}\left(y_{s}\right)^{-1}\right| .
$$

Now Propositions 2.3, 2.4 and Theorem 1.3 imply that there exist $c_{22}>0$, $c_{23} \geq 0$ and a $\Phi_{c_{22}}$-integrable random variable $Z$ not depending on $x$ such that for all $0 \leq t \leq T$,

$$
\left|\left[D \phi_{s t}(x)\right]^{-1}\right| \leq Z^{2} \exp \left(c_{23}\left(\ln ^{+}\left|y_{s}\right|\right)^{1 / 2}\right) .
$$

As in the proof of Theorem 2.1, we use the fact that there exists $\kappa \geq 0$ such that $\left|y_{s}\right| \leq \kappa^{2} Z^{2}\left(1+|x|^{2}\right)$. Hence

$$
\left|\left[D \phi_{s t}(x)\right]^{-1}\right| \leq Z^{2} \exp \left(c_{23}\left(\ln ^{+}\left(\kappa^{2} Z^{2}\right)\right)^{1 / 2}\right) \exp \left(c_{23}(2 \ln (1+|x|))^{1 / 2}\right)
$$

for all $0 \leq s, t \leq T$ and all $x \in \mathbf{R}^{d}$. Then (25) immediately implies (22).

It remains to prove (24).

Suppose first that $f: \mathbf{R}^{d} \rightarrow \mathbf{R}^{d}$ is a $C^{k}$-diffeomorphism for some $k \in \mathbf{N}$. Then for each $\alpha$ with $1 \leq|\alpha| \leq k, i=1, \ldots, d$ and for all $x \in \mathbf{R}^{d}$,

$$
D^{\alpha}\left(\left(f^{-1}\right)_{i}\right)(x)=\frac{p_{\alpha, i}\left(f^{-1}(x)\right)}{\left|\operatorname{det}\left[D f\left(f^{-1}(x)\right)\right]\right|^{n_{\alpha}}},
$$

where $n_{\alpha} \in \mathbf{N}$. In (26) $p_{\alpha, i}(y)$ is a polynomial in the partial derivatives of the components of $f$ up to order $|\alpha|$ evaluated at $y \in \mathbf{R}^{d}$. It is easy to verify (26) via induction on $|\alpha|$ using the chain rule and Cramér's rule.

For $0 \leq s, t \leq T$ and $x \in \mathbf{R}^{d}$ we have

$$
\phi_{s t}(x)=\phi_{0 t}\left(\phi_{0 s}^{-1}(x)\right) \text {. }
$$

Using the chain rule and (26) we see that for any $j \in\{1, \ldots, d\}$ we can write $D^{\alpha} \phi_{s t}^{j}(x)$ as a finite sum of a product of partial derivatives up to order $|\alpha|$ of components of $\phi_{0 t}$ and $\phi_{0 s}$ evaluated at $\phi_{0 s}^{-1}(x)$ multiplied by

$$
\left|\operatorname{det}\left[D \phi_{0 s}\left(\phi_{0 s}^{-1}(x)\right)\right]\right|^{-m}
$$

for some $m \in \mathbf{N}$. The fact that for any $d \times d$ matrix $A$ we have

$$
|\operatorname{det} A|^{-m}=\left|\operatorname{det} A^{-1}\right|^{m} \leq c_{24}\left|A^{-1}\right|^{d m},
$$

combines with Propositions 2.3, 2.4 and Theorem 1.3 to show that there exist $c_{25}>0, \gamma>0$ such that for all $0 \leq s, t \leq T$, all $x \in \mathbf{R}^{d}$ and all $1 \leq j \leq d$,

$$
\left|D^{\alpha} \phi_{s t}^{j}(x)\right| \leq \bar{Y} \exp \left(\gamma\left(\ln ^{+}\left|\phi_{0 s}^{-1}(x)\right|\right)^{1 / 2}\right)
$$

for some $\Phi_{c_{25}}$-integrable random variable $\bar{Y}$. Arguing as in the derivation of (22), we now obtain (24). 
3. Examples. We present two examples which show that the rates obtained in the first part of Theorem 2.1, and for the first derivative in Theorem 2.2 , are optimal, up to the constant $\gamma$. In fact we shall show slightly more, namely, that even if we fix $t>0$ and $s=0$, without taking the sup over $s$ and $t$, the rates specified in the theorems are still optimal up to the constant $\gamma$.

Since the first example is only a slight modification of Example 1 in [10], we shall only sketch it.

EXAMPLE 3.1. Let $d=1$ and fix $T>0$ and $0<\varepsilon<2$. For $2 \leq n \in \mathbf{N}$, define $\delta_{n}=\exp (n \sqrt{(2-\varepsilon) T \ln n})$ and $\gamma_{n}=\left(\delta_{n+1}-1+\delta_{n}\right) / 2$. Let $\sigma: \mathbf{R} \rightarrow \mathbf{R}$ satisfy:

(a) $\sigma \in C^{\infty}(\mathbf{R})$ and all derivatives of $\sigma$ are bounded;

(b) $\sigma(x)=x-\delta_{n}$ for $x \in\left[\delta_{n}, \gamma_{n}-1\right], 2 \leq n \in \mathbf{N}$;

(c) $\sigma\left(\gamma_{n}-x\right)=\sigma\left(\gamma_{n}+x\right)$ for $x \in\left[0, \gamma_{n}-\delta_{n}\right], 2 \leq n \in \mathbf{N}$;

(d) $\sigma(x)=0$ for $x \in\left[\delta_{n+1}-\frac{2}{3}, \delta_{n+1}-\frac{1}{3}\right], 2 \leq n \in \mathbf{N}$.

For $2 \leq n \in \mathbf{N}$, let $W_{n}$ be independent standard Brownian motions and define

$$
F(x, t)= \begin{cases}\sigma(x) W_{n}(t), & x \in\left[\delta_{n}-\frac{1}{2}, \delta_{n+1}-\frac{1}{2}\right], \\ \sigma(x) W_{2}(t), & x \leq \delta_{2}-\frac{1}{2} .\end{cases}
$$

It is easy to see that $F \in B_{u b}^{k, \delta}$ for any $k \in \mathbf{N}, 0<\delta \leq 1$. If $x \in\left[\delta_{n}, \gamma_{n}-1\right]$ and $\tau$ is the first time for which $\phi_{0 t}(x)$ hits $\gamma_{n}-1$, then

$$
\phi_{0 t}(x)=\left(x-\delta_{n}\right) \exp \left(W_{n}(t)-\frac{t}{2}\right)+\delta_{n},
$$

$0 \leq t \leq \tau$. There exists $n_{0}$ such that for $n \geq n_{0}$ we have $x_{n}=(e+1) \delta_{n} \in$ $\left[\delta_{n}, \gamma_{n}-1\right]$. A simple estimate shows that the sum over $P\left(\sup _{0 \leq t \leq T} \phi_{0 t}\left(x_{n}\right) \geq\right.$ $\left.\gamma_{n}-1\right)$ for $n \geq n_{0}$ diverges. Hence by the second Borel-Cantelli lemma we get

$$
\limsup _{n \rightarrow \infty} \frac{\sup _{0 \leq t \leq T} \phi_{0 t}\left(x_{n}\right)}{\gamma_{n}-1} \geq 1
$$

$P$-a.s. Furthermore,

$$
\lim _{n \rightarrow \infty} \frac{\gamma_{n}-1}{x_{n} \exp \left(\sqrt{(2-\delta) T \ln \ln x_{n}}\right)}=\infty
$$

for any $\delta>\varepsilon$. Hence, for any $\delta \in] 0,2[$ we have

$$
\sup _{x \geq 1} \sup _{0 \leq t \leq T} \frac{\phi_{0 t}(x)}{x \exp \left(\sqrt{(2-\delta) T \ln ^{+} \ln ^{+} x}\right)}=\infty
$$

$P$-a.s. Since $\sigma$ is symmetric around $\gamma_{n}$ on $\left[\delta_{n}, \delta_{n+1}-1\right]$, we also have

$$
\sup _{x \geq 1} \frac{\left|\phi_{0 T}(x)\right|}{x \exp \left(\sqrt{(2-\delta) T \ln ^{+} \ln ^{+} x}\right)}=\infty
$$

$P$-a.s. 
EXAMPLE 3.2. Let $d=1$. Let $\sigma: \mathbf{R} \rightarrow \mathbf{R}$ be a $C^{\infty}$-function with the following properties:

(a) $\sigma$ is periodic with period 1 ;

(b) $\sigma(x)=x$ for $-\frac{1}{4} \leq x \leq \frac{1}{4}$;

(c) $\sigma(x)=0$ for $\frac{3}{8} \leq x \leq \frac{5}{8}$.

Let $W_{n}, n \in \mathbf{Z}$, be independent standard Brownian motions and define

$$
F(x, t)=\sigma(x) W_{n}(t)
$$

for $n-\frac{1}{2} \leq x<n+\frac{1}{2}, t \geq 0$. Clearly $F \in B_{u b}^{k, \delta}$ for arbitrary $k \in \mathbf{N}, 0<\delta \leq 1$.

By Theorem 4.6.5 in [5], the associated flow $\phi$ is a flow of $C^{\infty}$-diffeomorphisms. Its spatial derivative satisfies for $n \in \mathbf{Z}, t \geq 0$,

$$
D \phi_{0 t}(n)=\exp \left(W_{n}(t)-\frac{1}{2} t\right)
$$

since the SDE based on $F$ is linear in a neighborhood of $n \in \mathbf{Z}$. Fix $T>0$ and $2>\varepsilon>0$. For $n \in \mathbf{N}$, define $\alpha_{n}=\sqrt{T(2-\varepsilon) \ln n}$. Then

$$
P\left(\exp \left(W_{n}(T)-\frac{1}{2} T\right) \geq \exp \left(\alpha_{n}\right)\right)=P\left(W_{n}(T) \geq \alpha_{n}+\frac{1}{2} T\right) .
$$

Because $W_{n}(T)$ is Gaussian with mean zero and variance $T$, there exists $c=c(T, \varepsilon)$ such that for all $n \in \mathbf{N}$,

$$
P\left(W_{n}(T) \geq \alpha_{n}+\frac{1}{2} T\right) \geq c \exp (-\ln n)=\frac{c}{n} .
$$

Using the Borel-Cantelli lemma, we see that for every $0<\delta<1$,

$$
\sup _{n \in \mathbf{N}} D \phi_{0 T}(n) \exp (-\sqrt{T(2-\delta) \ln n})=\infty
$$

$P$-a.s.

\section{REFERENCES}

[1] ARNold, L. and ImKelleR, P. (1996). Stratonovich calculus with spatial parameters and anticipative problems in multiplicative ergodic theory. Stochastic Process Appl. 62 19-54.

[2] ARNold, L. and ImkelleR, P. (1998). On the integrability condition in the multiplicative ergodic theorem. Stochastics Stochastics Rep. 64 195-210.

[3] BARLOW, M. and Yor, M. (1982). Semi-martingale inequalities via the Garsia-RodemichRumsey lemma, and applications to local times. J. Funct. Anal. 49 198-229.

[4] IMKELler, P. (1993). Occupation densities of Stratonovich stochastic differential equations with boundary conditions. Stochastics Stochastics Rep. 45 249-264.

[5] Kunita, H. (1990). Stochastic Flows and Stochastic Differential Equations. Cambridge Univ. Press.

[6] Ledoux, M. and Talagrand, M. (1991). Probability in Banach Spaces. Springer, Berlin.

[7] Mohammed, S. E. A. (1990). The Lyapunov spectrum and stable manifolds for stochastic linear delay equations. Stochastics Stochastics Rep. 29 89-131.

[8] Mohammed, S. E. A. (1992). Lyapunov exponents and stochastic flows of linear and affine hereditary systems. In Diffusion Processes and Related Problems in Analysis 2 (M. Pinsky and V. Wihstutz, eds.) 141-169. Birkhäuser, Boston.

[9] Mohammed, S. E. A. and Scheutzow, M. (1996). Lyapunov exponents of linear stochastic functional differential equations driven by semimartingales I: the multiplicative ergodic theory. Ann. Inst. H. Poincaré 32 69-105. 
[10] Mohammed, S. E. A. and Scheutzow, M. (1998). Spatial estimates for stochastic flows in Euclidean space. Ann. Probab. 26 30-55.

[11] Ocone, D. and PARDoux, E. (1989). A generalized Itô-Ventzell formula. Application to a class of anticipating stochastic differential equations. Ann. Inst. H. Poincaré 25 39-71.

INSTITUT FÜR MATHEMATIK

HUMBOLDT-UNIVERSITÄT ZU BERLIN

UNTER DEN LINDEN 6

10099 BERLIN

GERMANY

E-MAIL: imkeller@mathematik.hu-berlin.de
FACHBEREICH MatheMATIK, MA 7-5

TEChNISChe UnIVERSITÄT BERLIN

STRASSE DES 17 JUNI 135

10623 BERLIN

GERMANY

E-MAIL: ms@math.tu-berlin.de 